\title{
Effects of proton irradiations on GaN-based materials
}

\author{
J. P. Chang ${ }^{1}$, T. Y. Lin ${ }^{*, 1}$, H. F. Hong' ${ }^{2}$, T. C. Gunng ${ }^{2}$, J. L. Shen ${ }^{3}$, and Y. F. Chen ${ }^{4}$ \\ ${ }^{1}$ Institute of Optoelectronic Sciences, National Taiwan Ocean University, Keelung, Taiwan \\ 2 Institute of Nuclear Energy Research ROCAEC, LungTan, Taiwan \\ 3 Department of Physics, Chung Yuan Christian University, Chung-Li, Taiwan \\ ${ }^{4}$ Department of Physics, National Taiwan University, Taipei, Taiwan
}

Received 15 February 2004, accepted 5 July 2004

Published online 16 August 2004

PACS 61.82.Fk, 73.61.Ey, 78.55.Cr

We present the effects of proton irradiations on $n-G a N$ and InGaN/GaN light emitting diodes (LEDs) investigated by secondary ion mass spectrometry (SIMS), circular transmission line model (CTLM), Raman and photoluminescence (PL) measurements. The SIMS results show that the depth of proton implantation is nearly proportional to the energy of the hot protons. Proton implantation can result in the quenching in the luminescence and the degradation in the conductivity of $n-G a N$, which were found to be preserved to the temperature for device processing of $750{ }^{\circ} \mathrm{C}$. It was found that the intermixing of quantum wells (QWs) induced by proton radiations is negligible, and it is interesting to point out that InGaN/GaN QWs are more resistant to proton irradiations compared with the conventional III-V semiconductor QWs. Our results demonstrate the application of proton irradiations to the technology of electrical and optical isolation for the fabrications of compound semiconductor devices.

(C) 2004 WILEY-VCH Verlag GmbH \& Co. KGaA, Weinheim

\section{Introduction}

III-nitride semiconductors have been recognized as the very important materials for optoelectronic devices operating in the blue/UV region as well as for high temperature/high power electronic devices [1]. Proton irradiation has become important in the manufacture of a variety of devices, where it is used to produce semi-insulating regions for electrical isolation and also to change the refractive index for optical guiding $[2,3]$. The process of proton bombardment produces damage in the materials, which although required in certain applications can also have detrimental effects on device performance. So far, lots of studies are mainly focused on GaAs-based or InP-based materials[4, 5]. In this report, we present the effects of proton irradiations on $\mathrm{n}-\mathrm{GaN}$ and $\mathrm{InGaN} / \mathrm{GaN}$ LEDs.

\section{Experiment}

The n-GaN films doped with Si dopants were grown by metalorganic chemical vapor deposition on $c$ face sapphire substrates. A thin GaN buffer layer $(\sim 500 \AA)$ was deposited at $500{ }^{\circ} \mathrm{C}$ right before the epitaxial GaN $(\sim 2 \mu \mathrm{m})$ grown at $1000{ }^{\circ} \mathrm{C}$. The InGaN/GaN LEDs consist of an undoped series of ten periods of $2 \mathrm{~nm}$-thick $\mathrm{In}_{0.22} \mathrm{Ga}_{0.78} \mathrm{~N}$ wells and $9 \mathrm{~nm}$-thick GaN barriers grown on (0001) sapphire. The MQWs were sandwiched with a $4 \mu \mathrm{m} \mathrm{n}-\mathrm{GaN}$ layer and a $0.2 \mu \mathrm{m}$ p-GaN contact layer, which is the typical structure for light-emitting diodes. N-GaN samples were first implanted by proton with does of $1 \times 10^{15} \mathrm{p} / \mathrm{cm}^{2}$ and energy of $50 \mathrm{keV}$. Then, the implaned samples were thermally annealed at $550{ }^{\circ} \mathrm{C}$, $650{ }^{\circ} \mathrm{C}$ or $750{ }^{\circ} \mathrm{C}$ in $\mathrm{N}_{2}$ ambient for 10 minutes. The radius of circle of CTLM contact pads were $200 \mu \mathrm{m}$

\footnotetext{
*Corresponding author: tylin@mail.ntou.edu.tw
} 
in size and the spacings between the contacts pads were varied from 5 to $30 \mu \mathrm{m}$. Typical Ti $(21 \mathrm{~nm}) / \mathrm{Al}$ $(250 \mathrm{~nm})$ films were deposited by electron-beam evaporation, and the samples were rapidly annealed at $450{ }^{\circ} \mathrm{C}$ for 3 mins in a $\mathrm{N}_{2}$ ambient. The profile of implanted proton in $\mathrm{n}-\mathrm{GaN}$ was analyzed by SIMS measurements and simulated by TRIM software. Current-voltage data were taken at room temperature using a HP 4155A parameter analyzer. Additionally, InGaN/GaN LEDs were implanted by proton with does of $1 \times 10^{14} \mathrm{p} / \mathrm{cm}^{2}$ and energy of 100,200 or $300 \mathrm{keV}$, respectively. PL and Raman measurements were performed to investigate the optical properties of the implanted samples. A He-Cd laser with the wavelength of $325 \mathrm{~nm}$ was used as the excitation source for optical measurements.

\section{Results and discussion}

Figure 1(a) is the profile of implanted protons in $\mathrm{GaN}$ with different energies of $10 \mathrm{keV}, 50 \mathrm{keV}$ and $100 \mathrm{keV}$ measured by SIMS. It was found that the depth of proton is nearly proportional to the proton energy and is consistent with the TRIM simulation of the profile for proton implantation in n-GaN shown in Fig. 1(b). The depth of penetration of proton is estimated to be about $900 \AA$ per $10 \mathrm{keV}$ of proton energy.
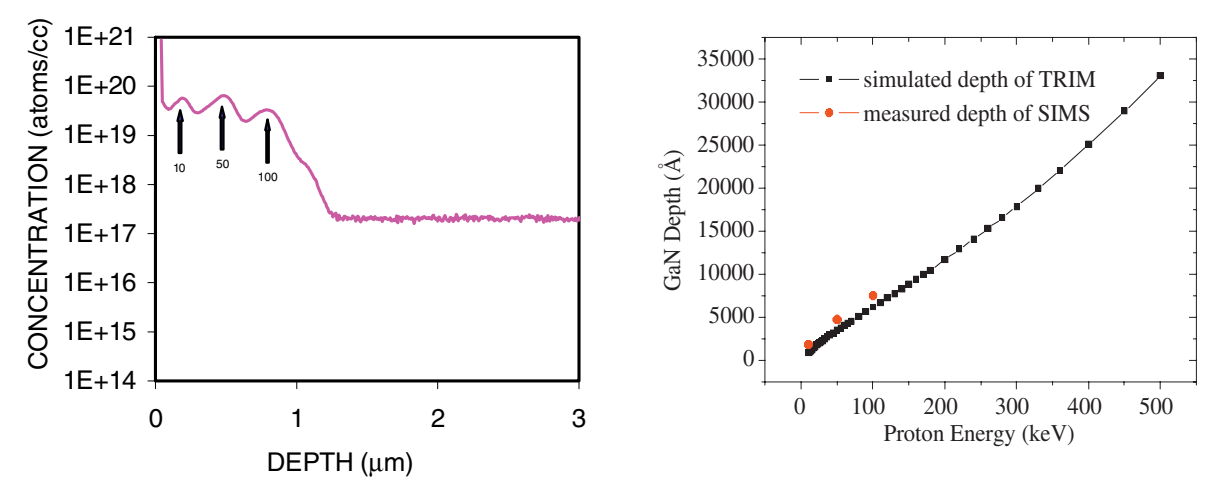

(a)

(b)

Fig. 1 (a) Distribution of proton with implantation energies of $10 \mathrm{keV}, 50 \mathrm{keV}$ and $100 \mathrm{keV}$ in $\mathrm{n}-\mathrm{GaN}$ measured by SIMS. (b) TRIM simulation of the profile for proton implantation in $\mathrm{n}-\mathrm{GaN}(\boldsymbol{\square})$ and implanted depths of proton measured by SIMS $(\bullet)$.

Figure 2 shows the room temperature PL spectra for the as grown, the $50 \mathrm{keV}$ proton implanted and the annealed-after-implantation n-GaN with does of $10^{15} \mathrm{p} / \mathrm{cm}^{2}$. The PL spectrun of the as grown sample shows a near band-edge emission centered at $363 \mathrm{~nm}$ and a defect-realted braod emission centered around $550 \mathrm{~nm}$, which are typical results for GaN films [6, 7]. From Fig. 2, it is clearly observed that the intensity of the band-edge emission is extensively reduced after proton irradiations in contrast with that observed in the as grown sample. It is believed that proton irradiations could produce the atomic displacement effect in $\mathrm{GaN}$ and generate extra structural defects, such as $\mathrm{V}_{\mathrm{Ga}}$ or $\mathrm{Ga}$ intersitials. Since the structural defects may increase more possible chances of nonradiative recombination, thus, the quenching of the band-edge emission induced by proton implantaions can be understood. Furthermore, it is found that the degradation in the optical properties of $\mathrm{GaN}$ by proton implanation is hardly to be recovered by thermal annealing up to the temperature for device processing of $750{ }^{\circ} \mathrm{C}$. Thus, it can be infered that the effects of proton irradiations on $\mathrm{GaN}$ is quite thermally stable.

In order to further investigate the influence of proton irradiations on the electrical properties of GaN, we performed the CTLM measurements. Figure 3 shows the specific contact resistance $\left(\rho_{\mathrm{c}}\right)$ as a function of the annealing temperature for $\mathrm{n}-\mathrm{GaN}$ irradiated by proton with does of $1 \times 10^{15} \mathrm{p} / \mathrm{cm}^{2}$ and energy of $50 \mathrm{keV}$. It should be emphasized that the specific contact resistance of unannealed implanted-GaN is too large to be measured by the present experimental set-up. Here, we therefore point out that proton irradia- 
tions can achieve the electrical isolation in GaN. Similarly, it is found that the degradation in the electrical properties of $\mathrm{GaN}$ by proton implanation is hardly to be recovered by thermal annealing at device processing temperature up to $750{ }^{\circ} \mathrm{C}$. From Fig. 3, the specific contact resistance is lowered with the increasing annealing temperature. This could be understood by recovery in crystal quality after annealing. However, the conducting properties of implanted-GaN become worse again at annealing temperature of $750{ }^{\circ} \mathrm{C}$. Thus, this conjectures the emergence of desorption of $\mathrm{n}-\mathrm{GaN}$ at temperature as high as $750{ }^{\circ} \mathrm{C}$.

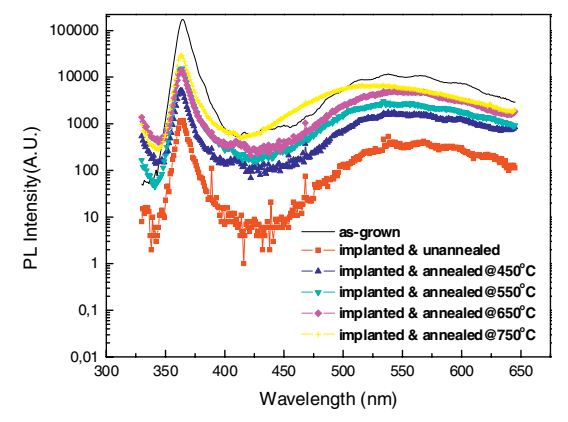

Fig. 2 Room temperature PL spectra for the as grown, the $50 \mathrm{keV}$ proton implanted and the annealed-after-implantation $\mathrm{n}-\mathrm{GaN}$ with dose of $10^{15} \mathrm{p} / \mathrm{cm}^{2}$. The annealing temperatures are $450{ }^{\circ} \mathrm{C}, \quad 550{ }^{\circ} \mathrm{C}, \quad 650^{\circ} \mathrm{C}$, and $750{ }^{\circ} \mathrm{C}$.

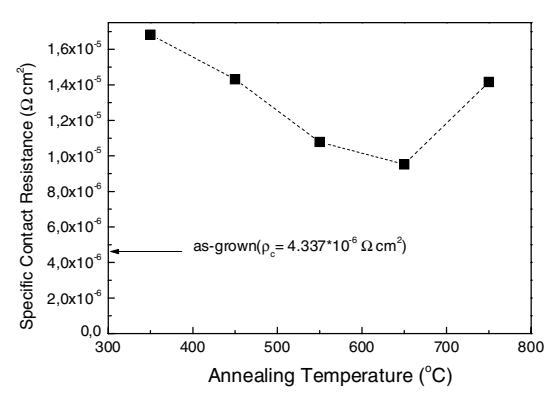

Fig. 3 Evolutions of the contact resistance in the proton-irradiated $\mathrm{n}-\mathrm{GaN}$ with $1 \times 10^{15} \mathrm{p} / \mathrm{cm}^{2}$, $50 \mathrm{keV}$ and annealed at $450{ }^{\circ} \mathrm{C}, 550{ }^{\circ} \mathrm{C}, 650{ }^{\circ} \mathrm{C}$ and $750{ }^{\circ} \mathrm{C}$, respectively.

Figure 4(a) shows the room-temperature PL spectra of the as-grown and the proton-irradiated InGaN/GaN LEDs. In the PL spectra, the as-grown sample shows only the band-edge emission around $2.7 \mathrm{eV}$. After proton implantation, the samples showed no evident shift in energy of band-edge emission, while no additional defect-related emission was found. Thus, the possible intermixing of QW induced by proton radiations is negligible with the protons at a dose of $1 \times 10^{4} \mathrm{p} / \mathrm{cm}^{2}$ up to the energy of $0.3 \mathrm{MeV}$. It also indicates that InGaN/GaN MQWs are more resistant to proton irradiations compared with the conventional III-V semiconductor QWs [8]. On the other hand, with the increase in the proton energy, the intensity of PL and Raman spectra both decrease and the full width at half maximum (FWHM) of PL and Raman spectra both increase. Figure 4(b) shows proton-energy dependence of the behaviors of the $A_{1}$ LO phonon mode of the InGaN layers in QWs. The irradiation-induced damages responsible for the
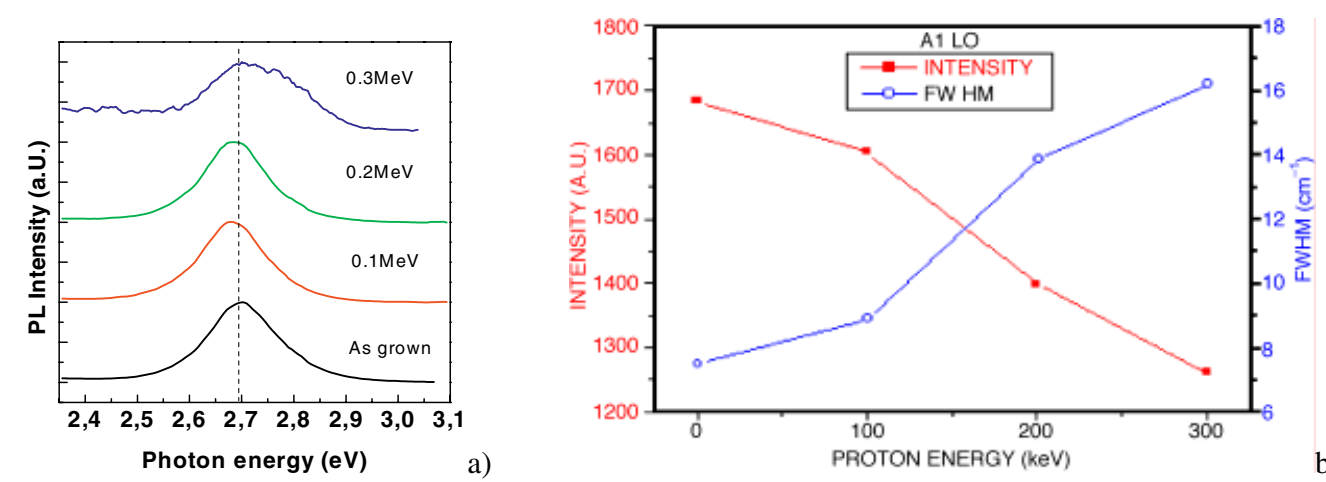

b)

Fig. 4 a) Room-temperature PL spectra of the as-grown and the proton-irradiated InGaN/GaN LEDs. The energy of the proton is indicated in the plot. (b) Proton-energy dependence of the intensity and the FWHM of the $\mathrm{A}_{1} \mathrm{LO}$ phonon mode of the InGaN layer 
electrical isolation in $\mathrm{GaN}$ has been attributed to the complexes of ion-beam-generated point defects with shallow donor or acceptor dopants [9]. The fact of the reductions in the intensity and broadening of the PL and Raman spectra are consistent with the degradation of crystallinity of the sample. The irradiationinduced damages may generate the nonradiative traps that can trap free carriers and reduce the intensity of band-edge emission of InGaN/GaN LEDs. Accordingly, we thus conclude that the proton irradiations help to the technology of electrical and optical isolation for the fabrications of GaN-based devices.

\section{Summary}

We have investigated the effects of proton irradiations on the electrical and optical properties of GaNbased materials. It is found that the effects of proton irradiations is quite thermally stable. The SIMS measurements show that the depth of proton distribution is nearly proportional to the energy of the implanted protons. Proton implantation can lead to the quenching in the luminescence and the degradation in the conductivity of $\mathrm{n}-\mathrm{GaN}$, which were found to be preserved to the device processing temperature up to $750{ }^{\circ} \mathrm{C}$. It was found that the intermixing of quantum wells (QWs) induced by proton radiations is negligible, and it is interesting to point out that $\mathrm{InGaN} / \mathrm{GaN} \mathrm{QWs}$ are more resistant to proton irradiations compared with the conventional III-V semiconductor QWs. Our results demonstrate the application of proton irradiations to the technology of electrical and optical isolation for the fabrications of III-nitrice semiconductor devices.

Acknowledgement This research was partially supported by the National Science Council of R. O. C.

\section{References}

[1] S. Nakamura, M. Senoh, S. Nagahama, N. Iwasa, T. Yamada, T. Matsushita, H. Kiyoku, Y. Sugimoto, T. Kozaki, H. Umemoto, M. Sano, and K. Chocho, Appl. Phys. Lett. 72, 211 (1998).

[2] P. L. F. Hemment, Application of Ion Beams to Materials (Inst. Phys. Conf. Lodon, 1975), Ser. No. 28.

[3] P. D. Townsend, J. C. Kelly, and N. E. W. Hartley, Ion Implantation, Sputtering and Their Application (Academic, New York, 1976).

[4] I. Danilov, J. P. de Souza, and H. Boudinov, J. Appl. Phys. 92, No. 8 (2002).

[5] H. Boundinov, J. P. de Souza, and C. Jagadish, Nucl. Instrum. Methods Phys. Res. B 175-177, 235 (2001).

[6] Shyam M. Khanna, Jim Webb, H. Tang, Alain J. Houdayer, and Cosmo Carlone, IEEE Trans. Nucl. Sci. 47, No. 6 (2000).

[7] H. M. Chen, Y. F. Chen, M. C. Lee, and M. S. Feng, J. Appl. Phys. 82, No. 2, (1997).

[8] H. C. Snyman and J. H. Neethling, J. Appl. Phys. 52, 6637 (1981).

[9] A. I. Titov and S. O. Kucheyev, J. Appl. Phys. 92, 5740 (2002).

[10] S. Yamaguchi, H. Kurusu, Y. Kawakami, S. Fujita, and S. Fujita, Phys. Rev. B 61, 10303 (2000). 